\title{
WHO WILL DECIDE ON FUNGAL NOMENCLATURE?
}

oncerns over fixing the application of scientific names, and limiting changes in names for non-scientific reasons, are widespread amongst biologists. Names are the one universal mechanism for correlating and communicating all categories of information on particular organisms. It is therefore critical that the application of names is regulated by pertinent international bodies. Next month in Shenzhen, China, a decision on what body should govern fungal nomenclature will be voted on.

One consequence of fungi traditionally being studied within botany, was that their naming was controlled by the International Rules [Code from 1952] of Botanical Nomenclature. The provisions in the Code are now considered and modified at subsequent International Botanical Congresses (IBCs), normally held at sixyear intervals. That was not seen as a major issue for mycologists while such congresses had a substantial mycological component and attendance. However, IBCs became ever larger and more expensive, mycologists a decreasing proportion of participants, and lacked enough symposia to cover the needs of a blossoming of mycological research into the 1960s. Mycologists felt increasingly marginalized in IBCs, and found their scale an impediment to personto-person interactions. As commented by Simmons (2010: 21), disillusionment set in as "mycology had outgrown the traditional relationship with botany, in both practical and scientific terms". In response to this situation, Geoffrey C. Ainsworth (19051998), who had retired in 1968 , initiated discussions to hold a separate International Mycological Congress (IMC). Having gained support from key mycological societies worldwide, this became a reality at Exeter (UK) in 1971.

It was recognized at IMC1 that improvements in the nomenclatural rules relating to fungi were needed, including issues over the separate naming of morphs in pleomorphic fungi, the acceptability of cultures as type material, and starting point dates. A Nomenclature Secretariat was established to pursue these matters, with Richard P. Korf(1925-2016) as Secretary. I recall Korf mentioning the idea of a separate Code for fungi at the inaugural meeting, but only as something not even to be considered; there was no dissent. The Secretariat tasked a series of committees with addressing the issues of concern, and they developed proposals to put to the 1981 IBC in Sydney, which were accepted at that Congress; the Secretariat was then dissolved, having completed its tasks. In general, IBCs have accepted proposals that were clearly supported by most mycologists, and particularly the Nomenclature Committee for Fungi (NCF).

Nomenclatural matters have become an increasingly important aspect of IMCs, initially with debates on topics of concern, but since IMC9 in Edinburgh in 2010 have incorporated Nomenclature Sessions and questionnaires circulated to all Congress delegates. At IMC10, and subsequently at meetings in Amsterdam the following year, mycologists supported the transfer of decision making on issues only concerning fungi from IBCs to IMCs. This was discussed at the IBC held in Melbourne later in 2011, when a Special Subcommittee was formed to address how this should be done. That Committee has now reported, and the proposals made are to be voted on at the IBC in Shenzhen in July 2017.

Key mycological committees support the proposals made (see pp. (9)-(11) in this issue), but there is a concern they may not be accepted next month. The Nomenclature Section meetings at the IBC in Vienna in 2005 and Melbourne in 2011 were attended by just seven and ten mycologists, out of a total of 198 and 204 delegates, respectively. In contrast, at IMC10 in Bangkok in 2014, 74-94 mycologists attended the Nomenclature Sessions, and 117 turned in ballot papers.

At present mycologists do not favour leaving the current Code, re-named the International Code of Nomenclature for algae, fungi, and plants in 2011. They appreciate the care with which the rules are refined by botanists well-versed in the intricacies of nomenclature facing the same problems as mycologists, for example on issues related to electronic publication. At the same time, mycologists are uncomfortable with, and do not wish, decisions on matters relating soley to fungi to continue to be taken at IBC meetings when so few mycologists are present, and the Nomenclature Committee for Fungi (NCF) is elected. There is also a feeling that a six year interval between possible changes

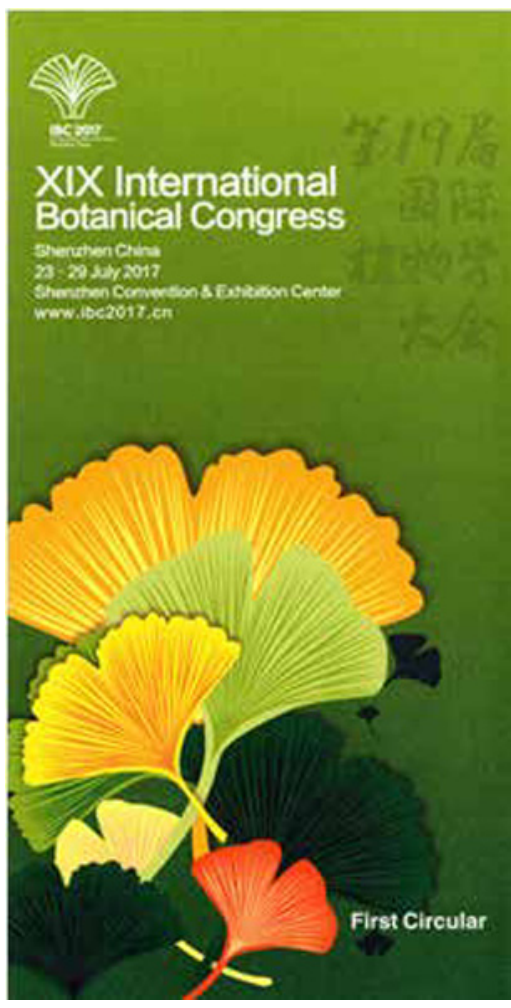

is too long when aspects of the subject are having to adapt to new approaches. As there will be two IMCs before the next IBC in 2023, this will lead to two decision-making opportunities before the next IBC.

All mycologists unable to attend the Shenzhen meetings and who work in institutions that have institutional votes to cast are urged to encourage their representatives to support the proposals for a change in the control of matters relating specifically to fungi from IBCs to IMCs. If these proposals do not gain approval next month, it is unlikely that mycologists would be content with the issue being referred to another committee, charged with reporting at the 2023 IBC. IMC11 in Puerto Rico in 2018 would then face the challenge of deciding whether to reject that decision and operate independently - a schism I would not wish to see.

Simmons EG (2010) The Interntaional Mycological Association: its history in brief with summaries of its International Mycological Congresses and diverse international relationships. IMA Fungus 1: $18-100$

David L. Hawksworth Editor-in-Chief, IMA Fungus (d.hawksworth@kew.org) 